\title{
El camino del amor en el Banquete: un ascenso desviado a lo divino*
}

\author{
The path of love in the Symposium: a deviated ascent to the divine
}

Por: Abril, Sain*

UBA

Buenos Aires, Argentina

Email: abrilsain@gmail.com

Fecha de recepción: 03/07/2021

Fecha de aprobación: 15/07/2021

DOI: https://doi.org/10.30972/nvt.1725708

\section{Resumen}

En el presente trabajo me propongo dar cuenta del carácter del camino del amor a lo que es en sí en el Banquete de Platón. Para esto partiré de una lectura recíproca entre todas las voces del simposio, alejándome de la interpretación que prioriza únicamente el discurso de Sócrates-Diotima. Entonces buscaré mostrar que en el Banquete hallamos un modelo antropológico de unidad entre el cuerpo y el alma, con lo cual el aspecto sensible resulta también útil a la búsqueda filosófica. Procuraré fundamentar esto doblemente: mostraré que (1) los pasos en el camino de la scala amoris implican no solo un ascenso vertical sino también un desvío necesario hacia el carácter horizontal de la inmanencia; y (2) que el avance vertical, es decir el continuo ascenso, no implica un desprecio de los pasos anteriores sino más bien un desapego

\footnotetext{
* Este trabajo fue realizado en el marco de mi Tesis de Licenciatura, en la cual he trabajado en mayor profundidad y detalle el diálogo platónico Banquete y las temáticas aquí discutidas. Siguiendo la misma línea de interpretación que en mi Tesis, desarrollo aquí con mayor amplitud un análisis particular de la scala amoris descripta por Diotima en el discurso de Sócrates.

* Profesora y Licenciada en Filosofía por la Universidad de Buenos Aires. Actualmente se encuentra estudiando filosofía platónica en el marco de un Doctorado en Filosofía en la misma universidad, donde además participa del programa de extensión "Filosofía y Territorio" y de un grupo de estudio e investigación UBACYT dirigido por la Dra. María Angélica Fierro acerca de la filosofía clásica.
} 
que desliga al amante de los lazos esclavizantes con los objetos particulares de su amor, sean estos corporales o espirituales.

Palabras clave: Platón- Banquete- Dualismo- Amor- Cuerpo

\section{Abstract}

Here I intend to show the nature of the path of love of wisdom in Plato's Symposium. My starting point will be a reciprocal reading among all the voices in the symposium, moving away from the interpretation that prioritizes only the SocratesDiotima speech. Then I will try to show that in the Symposium we find an anthropological model of unity between the body and the soul, with which the sensitive aspect is also considered useful for the philosophical search. I will support this thesis in a double way: I will show that (1) the scala amoris steps imply not only a vertical ascent but also a necessary deviation towards the horizontal character of immanence; and (2) that the vertical ascent does not imply a disregard for the previous steps but rather a detachment that frees the lover from the enslaving ties which bonds them to the particular objects of their love, be they corporeal or spiritual.

Keywords: Plato- Symposium -Dualism- Love- Body

\section{Cómo citar este artículo:}

APA: Sain, A. (2021). El camino del amor en el Banquete: un ascenso desviado a lo divino. Nuevo Itinerario, 17 (2), 1-26. Recuperado de: (agregar dirección web) 
Nadie habrá dejado de observar que con frecuencia el suelo se pliega de manera tal que una parte sube en ángulo recto con el plano del suelo, y luego la parte siguiente se coloca paralela a este plano, para dar paso a una nueva perpendicular, conducta que se repite en espiral o en línea quebrada hasta alturas sumamente variables. [...]Agachándose y poniendo la mano izquierda en una de las partes verticales, y la derecha en la horizontal correspondiente, se está en posesión momentánea de un peldaño o escalón. Cada uno de estos peldaños, formados como se ve por dos elementos, se sitúa un tanto más arriba y adelante que el anterior, principio que da sentido a la escalera, ya que cualquiera otra combinación producirá formas quizá más bellas 0 pintorescas, pero incapaces de trasladar de una planta baja a un primer piso.

Julio Cortázar, «Instrucciones para subir una escalera» (1962, p. 11)

\section{Introducción}

El Banquete ${ }^{1}$ de Platón se nos despliega como un diálogo bien temperado, armonizado a la manera de una pieza musical. En este alegre encuentro en la casa de Agatón, los invitados se entretienen elogiando cada uno a su turno al dios del Amor ${ }^{2}$.

\footnotetext{
${ }^{1}$ Todas las citas de las fuentes son del Banquete a menos que se indique lo contrario, antecediendo la referencia con las abreviaturas usuales de los diálogos platónicos según el LSJ. Las traducciones del Banquete corresponden siempre a Ludueña (2015), a menos que se indique lo contrario.

${ }^{2}$ Los discursos del Banquete versan sobre Érós. Algunos autores eligen no traducir el término debido a la complejidad de su significado, los hay quienes optan por la opción admitida por la Real Academia Española «eros», y finalmente quienes lo traducen como "amor» (existe un acuerdo en que la mayúscula refiere al dios Éros y la minúscula a aquello que el dios causa, el éros, aunque en Platón no estaba esta distinción gráfica y las ocasiones en las que se refiere a uno u otro pueden variar de acuerdo a la interpretación). En este caso optaremos por "amor» cuando nos referimos a la emoción y «Amor» cuando se trata del "dios» (theós) o "divinidad» (daímon). Ha de considerarse siempre, sin embargo, que el término remite al griego érós, noción compleja que muchas veces refiere a la vez a la emoción y a la divinidad (Dover, 1980), y en Banquete particularmente adquirirá un carácter intermediario (metaxý) entre lo divino y lo humano. Así, como lo expresara ya Robin (1908, pp. 172-173), al ubicarse Érós entre lo humano y lo divino puede vincular ambos planos, puesto que se produce una síntesis de cualidades
} 
Así, el primer momento del diálogo desarrolla una instancia de competencia lúdica alrededor de la consigna del encomio a la divinidad. El segundo momento es instaurado por el quiebre que se produce cuando Sócrates declara expresamente que no continuará con el elogio según se venía haciendo, y propone en cambio decir la verdad acerca del Amor. Y, finalmente, el tercer y último momento es ilustrado por la repentina e inesperada aparición de Alcibíades, quien, ebrio y con actitud festiva, promete también la verdad, pero esta vez no acerca del dios sino acerca de su particular enamorado: Sócrates.

A partir de este ordenamiento, el Banquete se ha leído tradicionalmente como un simposio puramente intelectual. Es decir, se ha entendido generalmente que la intención de Sócrates de terminar con la dinámica anterior de los elogios previos echaba por tierra estos discursos, y que habría que prestar especial atención únicamente a su participación. En este sentido, las interpretaciones más clásicas consideran el discurso de Sócrates como el lugar en el diálogo donde deberíamos ir a buscar el pensamiento de Platón, allí donde oímos de parte del filósofo las palabras de la sabia sacerdotisa de Mantinea. Para Chen (1983), por ejemplo, de todos los discursos, el de Sócrates es filosóficamente el más importante, y la inclusión de Diotima es en realidad la propia teoría de Sócrates en el diálogo (p. 66). Por otra parte, muchos autores saltean el análisis de los primeros discursos para encargarse únicamente del de Sócrates. Este es el caso de Cornford (1974), quien afirma: «He de pasar de largo los primeros discursos que esbozan distintas sugerencias en torno a la naturaleza de Eros, las cuales son recogidas o criticadas en el discurso de Sócrates» (p. 133) ${ }^{3}$. De estas mismas lecturas se sigue también que el cuerpo -el cuerpo del amante que avanza en su camino, sus sentidos perceptivos físicos, y también el cuerpo del amado, y los demás cuerpos bellos que funcionan como objeto del amor - tiene un rol negativo en la actividad filosófica, puesto que estas privilegian el instante culminante de acceso a la Idea de la belleza respecto a los peldaños anteriores. De la misma manera en que, al atender especialmente al discurso de Sócrates-Diotima los

contrarias en él - una naturaleza sintética - que le posibilita la conexión de ámbitos que se hallan separados.

${ }^{3}$ En esta línea se inscriben también las lecturas de Robinson (1998), Vernant (2001), Reale (2010), o Boeri (2016), entre otros. 
primeros elogios quedan relegados a las palabras de la filosofía y la sabiduría, el lugar del cuerpo en el camino amoroso revelado a Sócrates queda olvidado en los primeros pasos de la iniciación en pos de atender especialmente al alma, en la convicción de que es esta únicamente la que guiará al filósofo correctamente. Así, por ejemplo, Dover (1980) asegura que para el Sócrates del Banquete el amor excitado por los cuerpos bellos es «simplemente» el primer paso hacia la satisfacción del deseo del alma por la belleza trascendental (p. 2). Vernant (2001, pp. 149-165), por su parte, sostiene que la posición de Platón en el Banquete aparece únicamente en el discurso de SócratesDiotima, y que en este discurso el amor actúa verticalmente en dirección al cielo, dirección que, según describe el autor, hace rápidamente a un lado al cuerpo, el cual debe ser despreciado en la medida en que se avanza camino a la Idea de belleza ${ }^{4}$.

Los movimientos de interacción de los lógoi en el Banquete, sin embargo, deberían mínimamente hacernos sospechar de esta interpretación unívoca. La lectura de la cual aquí partimos pretende en cambio atender a la riqueza de todas las voces del simposio, de una manera más bien perspectivista ${ }^{5}$. Si bien es cierto que el discurso de Sócrates es el único que logra desplegar cabalmente las distintas claves que Platón proporcionó en los discursos previos ${ }^{6}$, esto no significa que los primeros elogios no nos ofrezcan ninguna información relevante sino, por el contrario, manifiestan un ordenamiento particular al que hay que prestar cuidadosa atención, así como la iniciación amorosa de Diotima debe seguirse en el orden adecuado para resultar

\footnotetext{
${ }^{4}$ La idea de desprecio al cuerpo en el Banquete está sostenida también por la interpretación más general de que Platón tiene una consideración negativa de lo sensible para el conocimiento basada en diálogos donde esto puede verse mayormente subrayado, como es el caso del Fedón o algunas secciones del Fedro. En estos lugares de la obra platónica lo más sensato para el filósofo parece ser despreciar el cuerpo y emprender la búsqueda de la sabiduría solo con su alma, pues cuando esta última «intenta examinar algo en compañía del cuerpo, está claro que es engañada por él» (Phd. 65c). Es relevante señalar que el contexto dramático del Banquete acompaña un escenario más ameno para con lo sensible que el Fedón, momento angustioso por la inminente muerte de Sócrates. En las líneas que destacan la situación dramática particular de cada diálogo en vinculación con su contenido filosófico encontramos a Ludueña (2015), Rowe (2007), Ferrari (1987), Nussbaum (2001 [1986]), Cornford (1974), y Taylor (1926).

${ }^{5}$ Soares (2012, pp. 13-22) desarrolla una lectura perspectivista del Banquete según la cual Platón hace interactuar en este diálogo distintas perspectivas a través de los géneros discursivos encarnados por cada personaje, con lo cual Platón procura explicitar la complejidad del problema erótico.

${ }^{6}$ Fierro (2019, p. 192), coincidiendo en la atención que requieren todas las intervenciones de los personajes en el simposio, propone que el discurso de Sócrates es el único que logra comprender cabalmente los puntos esenciales que Platón presenta en los discursos previos.
} 
exitosa. La autora Peixoto $(2012$, p. 178) propone una bella figura para acercarse al Banquete. Ella explica que la experiencia que ofrece este diálogo se presenta como un caleidoscopio. A través de este peculiar artefacto podemos ver diversas imágenes que se reorganizan paso a paso en cada discurso, como una experiencia óptica en la cual se va despertando una operación intelectiva a medida que se van ordenando y desordenando los vidriecitos de colores y avanzamos en nuestra lectura.

Partiendo de aquí, el propósito de este trabajo es dar cuenta del carácter del camino del amor en el Banquete atendiendo a todas sus voces ${ }^{7}$. Mi hipótesis es que en este diálogo Platón propone un modelo antropológico de unidad entre el cuerpo y el alma, con lo cual el aspecto sensible resulta también útil a la búsqueda filosófica de lo que es en sí ${ }^{8}$. Procuraré fundamentar esto doblemente:

(1) Mostraré que los pasos en el camino de la scala amoris implican no solo un ascenso vertical sino también un desvío necesario hacia la horizontalidad de la inmanencia y lo corporal.

En un primer apartado, utilizando la imagen de una escalera, plantearé la posibilidad de que cada peldaño en la scala amoris de Diotima tenga un valor tanto por su verticalidad como por su horizontalidad, incluyendo con esto no solo el ámbito «celestial», del alma que busca la virtud y se dirige hacia arriba, sino también la cara «terrenal» del camino, el ámbito del cuerpo y del mundo. Esta forma doble del peldaño es la que hace patente la necesidad de un desvío en la continuidad vertical del ascenso, es decir, el requerimiento de tornar horizontal el camino una y otra vez para volver a la cotidianeidad que nos rodea. Al mismo tiempo, esta figura nos permitirá pensar la inclusión de dos ámbitos aparentemente opuestos, como lo son lo vertical y

\footnotetext{
7 Este punto de partida va de la mano con un enfoque heterodoxo del texto como el que propone Dixsaut (2001), quien invita a abordar los diálogos platónicos — muchos de los cuales ella misma ha traducido y comentado- desde un lugar deconstructivo que implica ante todo una manera de filosofar. Esto se aleja de la pretensión de un conocimiento objetivo o doctrinal del texto, pretendiendo más bien un esfuerzo en comprender los sentidos problemáticos de los términos (Dixsaut, 2001, p. 12). En un enfoque similar se encuentra la lectura de Annas (2003).

${ }^{8}$ Interpretaciones similares podemos hallar en Cornelli (2016), Fierro (2013*), Peixoto (2012) y Broadie (2001). Peixoto (2012, pp. 171-172), por mencionar un ejemplo, señala al ser el primer movimiento del camino filosófico el dirigirse hacia los cuerpos bellos, el discurso de Diotima presupone que es la naturaleza sensible del objeto la que es capaz de suscitar el interés por lo bello, esto es, la que tiene la capacidad de provocar el movimiento inicial, en palabras de la autora, de romper el estado de inercia generando un paso fundamental para que el proceso comience. Por esta razón, el rol del cuerpo en el camino del amor tiene una intervención más relevante que la de mero paso a superar.
} 
lo horizontal, y abrazarlos en un mismo propósito con la fuerza del amor, que funciona siempre como puente intermediario de los extremos.

(2) Mostraré que el avance vertical, es decir el continuo ascenso, no implica un desprecio o rechazo de los elementos y pasos anteriores sino más bien un desapego que desliga al amante de los lazos esclavizantes con los objetos particulares de su amor, sean estos corporales o espirituales.

En un segundo apartado, para explicar cómo es posible avanzar en el ascenso al tiempo que se retorna constantemente al nivel horizontal de lo corporal sin caer por esto necesariamente en una lectura despreciativa, desarrollaré la noción de «desapego». Esta idea me permitirá ilustrar el resultado práctico de una comprensión que lleva a debilitar los lazos obsesivos que tienen al amante preso de su amor, tanto en cuerpo como en alma. El desapego libera al amante de una relación particular y especial, y le otorga en cambio la posibilidad de expandir su amor libremente. De esta forma, el amante que avance en su camino comprendiendo gradualmente la universalidad de la belleza, volverá constantemente al encuentro con los cuerpos y los deseos del cuerpo, y deberá actualizar su ejercicio de desapego para poder relacionarse con todos los aspectos de la vida sin perder de vista la dirección de su andar.

\section{La forma del peldaño}

En el discurso de Sócrates-Diotima aparece la necesidad de una suerte de interrupción en el orden ascendente, a lo cual me referiré como un necesario «desvío» en el camino amoroso. Buscaré exponer aquí que la iniciación de la sabia sacerdotisa incluye un constante desvío en su verticalidad ascendente en donde se vuelve relevante el rol del cuerpo en el quehacer filosófico. A partir de la lectura que aquí proponemos, procuraré además mostrar que este desvío se extiende e incluye de manera armónica al resto de los discursos del diálogo.

Como leemos en el sexto discurso, Diotima le ha enseñado a Sócrates la manera adecuada de seguir el camino del amor, subrayando en varias ocasiones que los pasos se deben seguir «correctamente» si se pretende llegar a la contemplación de 
lo divino. Así, desde que comienza la «revelación última» en 210a hasta el fin de la participación de Diotima en 212 a, el término orthõ̃s aparece nada menos que seis veces:

Ahora bien, no sé si podrás ser iniciado en la revelación última, la visión suprema gracias a la cual, si uno procede correctamente (orthõs), aquellas [enseñanzas] cobran sentido. Pero aún así te la revelaré. [...] Es preciso dijo- que quien avance correctamente (orthõs) en esta empresa comience ya de joven a acercarse a los cuerpos bellos y que, si el guía lo conduce correctamente (orthõs), primero se enamore de un único cuerpo. (210a)

Quien haya sido guiado en el terreno erótico hasta este punto, tras contemplar en orden y correctamente (orthōs) las cosas bellas [...] advertirá de pronto algo asombroso, bello por naturaleza. (210e)

Así pues, siempre que elevándose por sobre las cosas que nos rodean mediante la pederastia correcta (orthōs) - alguien comienza a advertir este algo bello [del que hablo], ya casi está alcanzando la perfección. En efecto, este es el camino correcto (orthōs) en las cosas de Eros. $(211 b-c)^{9}$

Esta constante insistencia por parte de Platón en la utilización del término orthõ̃s nos llama a detenernos a analizar su sentido. Después de todo, como señala acertadamente Ludueña (2015), "La lectura frecuente del Banquete revela que nada en él es accidental» (p. 102) ${ }^{10}$. El adverbio orthõ s proviene del adjetivo orthós, que significa «derecho», «de pie», «recto», «correcto», «sólido», «verdadero»; el sustantivo homónimo significa a su vez «línea recta», "camino recto», y de allí orthótes, «posición vertical», «rectitud». Es decir, Diotima pretende describir un camino recto y sólido que, solo de esta manera, permitirá el ascenso a la verdad. La idea de una rectitud que se eleva nos hace pensar desde el principio en el objetivo final

\footnotetext{
9 Traduje en todos los casos «correctamente» / «correcto/a» si bien en algunas ocasiones Ludueña (2015) opta por «adecuadamente» / «adecuado/a» para mostrar más claramente la constante aparición del mismo término.

${ }^{10}$ En una lectura similar, Rowe (1998, p. vii) sostiene que el Banquete es, por sobre todo, una cuidadosa obra de arte. Por otro lado y contra Rowe, la interpretación de Ludueña que aquí traemos apoya también la lectura perspectivista, al subrayar que en la minuciosa y deliberada escritura de Platón yace «la imposibilidad de concentrarse solo en el discurso de Sócrates como si allí estuviera la cifra del pensamiento de Platón» (2015, p. 102).
} 
que se persigue -la contemplación de la Belleza en sí- que nos atrae desde arriba y nos invita a elevarnos más y más hasta alcanzarlo. Cada paso que el amante avance servirá de escalón para seguir ascendiendo, y es por ello por lo que es necesario que el camino se realice de la manera en que Diotima lo describe, y no de otra, es decir que se siga "correctamente». Platón hace hincapié en este respecto, en que no hay otra manera de recorrer el camino amoroso si no es en línea recta y hacia arriba ${ }^{11}$.

Si bien estas pistas parecieran indicarnos que no se pueden alterar ni saltear pasos en el camino del amor, es innegable al mismo tiempo que Platón llama nuestra atención con otras señales menos ordenadas, más caóticas. Siguiendo con la observación de Ludueña (2015), el autor nos advierte: «Imposible es también concentrarse solo en lo que los personajes dicen» (p. 102). En 185c-d, Platón altera el orden de los elogios provocándole a Aristófanes un ataque de hipo que le impide hablar en su lugar. Entonces el médico Erixímaco promete curarle el hipo, haciendo valer su técnica y su nombre - Erixímaco significa literalmente - «el que combate el hipo»- y hablar además en su turno hasta que se calme.

Con esto, el orden se ve trastocado e interrumpido. Pero, además, Platón no abandona el juego del caos hasta que a Aristófanes le toca hablar nuevamente, cuando sentencia acerca de su ataque de hipo: «iY se calmó del todo! Aunque no antes de aplicar el estornudo; cosa que me asombra: esto de que la parte "moderada" del cuerpo desee estrépitos y cosquilleos tales como un estornudo» (189a). Con este breve comentario al pasar, Platón explicita la necesidad del desorden para retornar al orden, como lo es el estornudo a la cura del hipo, al tiempo que introduce al cuerpo

\footnotetext{
${ }^{11}$ Si bien no abundaremos aquí en la intertextualidad con otros diálogos de Platón, es difícil no pensar con respecto a este punto en la alegoría de la línea de República $509 \mathrm{~d}$ y ss. Allí también se describe un camino recto - literalmente una línea - que se encamina hacia arriba, hasta el principio de todo. En ambos casos se comienza por lo sensible y luego se avanza a lo inteligible, ayudándose de los pasos previos como si fueran «escalones» (epíbasis en R. 511b, epanabasmós en Smp. 211c). Además, en la República, el método dialéctico que se podría identificar en tal diálogo con la filosofía realiza una «labor de atracción», "atrayendo» al ojo del alma hacia el principio mismo (R. 533c-d). Si bien en la República no se hace referencia a la Idea de Belleza en este punto, aparece sin embargo la noción de atracción que en el Banquete hallamos en relación con lo bello. En la República asimismo se plantea la posibilidad de que exista una «musa filosófica» que guíe a los hombres a gobernar con justicia, lo cual es descripto como una «inspiración divina» que no es otra cosa que un "verdadero amor» $(R .499 \mathrm{~b}-\mathrm{c})$. Por lo cual podemos pensar que en ambos diálogos de madurez Platón estaba pensando en un camino amoroso para alcanzar las Ideas, camino que comienza siempre por lo sensible, y se sirve de esta base tangible para escalar sobre ella hacia lo más inteligible.
} 
con un protagonismo principal que irrumpe en la anterior armonía y continuidad dramáticas. Es decir que encontramos ahí, en tensión con la idea de lo correcto y ordenado del discurso de Sócrates-Diotima, la aparición de lo inesperado y lo caótico, y la necesidad del desorden, esto es, de un desvío para retomar el camino correcto ${ }^{12}$.

Otro de los llamados de atención de Platón que pone en tensión la idea de orden descripta en la iniciación de Diotima es la estrepitosa aparición de Alcibíades hacia el final, borracho y acompañado de música y más amigos:

Pero de pronto (exaíphnes) unos golpes en la puerta del patio produjeron un gran alboroto, como el de una pandilla de juerguistas; y se oyó la melodía de una flautista. [...] Y, según Aristodemo, poco después se oyó en el patio la voz de Alcibíades que, totalmente ebrio y gritando muy alto [...] se detuvo junto a la puerta coronado con una espesa corona de hiedras y violetas y con muchísimas cintas alrededor de la cabeza. (212c-d)

Alcibíades irrumpe en la casa de Agatón «de repente». El término griego es el adverbio exaíphnes que significa que algo ocurre de pronto e inesperadamente. Este adverbio es utilizado cuatro veces en todo el diálogo y, como es de esperar, no azarosamente $^{13}$. La última mención del término es hacia el final del discurso de Alcibíades cuando leemos:

12 Existen varias interpretaciones que entienden el ataque de hipo de Aristófanes como una representación graciosa del personaje que encarna el comediógrafo en el diálogo. En esta línea se inscribe, por ejemplo, el pensamiento de Nussbaum (2001 [1986], pp. 171-176), quien considera que el episodio del hipo de Aristófanes no es más que un efecto cómico, o el de Rowe $(1998$, p. 146) quien afirma que tanto el discurso de Aristófanes como su ataque de hipo forman parte de la caracterización platónica de un personaje al que no le interesan más que los aspectos físicos de la vida. Entre otras de las muchas interpretaciones que se han propuesto para explicar el ataque de hipo (vid. Rosen, 1968, pp. 90 y ss.) destacamos las siguientes: i) que se trata de una venganza de Platón al Aristófanes histórico quien, en Las Nubes, había ridiculizado a Sócrates (Brochard, 1940, pp. 42-81); ii) que se trata de una ocurrencia dramática de Platón a quien le habría parecido divertido incluir este episodio debido al significado del nombre Erixímaco - «el que combate el hipo» (Guthrie, 1975, p. 382); iii) como un artilugio de Platón para alterar el orden dialéctico de los discursos, ya sea para acercar a los discursos de Pausanias y Erixímaco que son similares (Gierse, 1970, pp. 49-76) o acercar al poeta cómico y al poeta trágico (Isenberg, 1940), o bien para conseguir una unidad armónica entre los primeros cuatro discursos, en la que el elogio de Fedro representaría la unidad, los de Pausanias y Erixímaco la dualidad, y el de Aristófanes, la tríada, símbolo de la totalidad en las cosmogonías antiguas (Hoffmann, 1947). Sin desestimar estas propuestas, nuestra lectura aquí pretenderá darle un sentido más pleno de contenido filosófico a la intervención del ataque de hipo, según los indicios que hallamos en la propia fuente.

${ }^{13}$ El primer uso del término en el diálogo se da hacia el final de la descripción iniciática de Diotima acerca de los asuntos amorosos, cuando acontece la visión de la Belleza en sí de repente (210e). La segunda vez que Platón escribe exaíphnes es para anunciar la llegada de Alcibíades, y luego cuando este, 
Entonces Agatón se levantó para ir a acostarse al lado de Sócrates, pero de pronto (exaíphnes) -me contó Aristodemo- un buen número de juerguistas llegaron a las puertas de la casa [...]. Todo se llenó alborotó. Y ya en medio del desorden general (oukéti en kósmo), los forzaron a beber enormes cantidades de vino. (223b)

Con estas pistas de que la rectitud muchas veces se quiebra dejando lugar al desorden y lo espontáneo, nos focalizaremos ahora en particular en el significado de este desvío en las enseñanzas de la sabia sacerdotisa.

Para representar la iniciación erótica de Diotima en el discurso de Sócrates, usaré la imagen de una escalera. Pero tal vez sea útil además considerar, como lo ilustra Cortázar, la naturaleza de este instrumento. En efecto, la escalera está compuesta de escalones, y cada escalón está formado por dos elementos, una parte horizontal y una vertical. Con esta configuración, al situarse uno un tanto más arriba y más adelante que el anterior, dan sentido a la figura que permite ascender. Igualmente, el camino amoroso tiene esta ordenación: es vertical, en tanto se dirige hacia arriba, pero no en una continua línea recta sino que está conformado por escalones, cada uno de los cuales incluye también una parte horizontal que una vez transitada revela su aspecto vertical y permite elevarse hasta el siguiente peldaño ${ }^{14}$.

Teniendo en mente esta figura, podemos imaginar ahora que mientras el aspecto vertical de cada peldaño evoca todo lo celestial y lo más sutil que se eleva, las partes horizontales nos remiten a lo terrenal y tangible, esto es, al aspecto inmanente de la vida diaria (recuérdese aquí el episodio del hipo y la inesperada aparición de Alcibíades y sus amigos). Esta configuración doble se hace patente ya desde el primer peldaño de la scala amoris, donde la indicación es ir hacia un cuerpo pero también -

de repente, ve a Sócrates (213c). La última aparición de exaíphnes es con la llegada de muchos otros amigos (223b). Para un análisis del uso de este término en Banquete, véase por ejemplo Velázquez (1986, pp. 75-76) o Soares (2009, p. 102).

${ }^{14}$ En una lectura distinta a la nuestra hallamos la de Chen (1983), cuya interpretación indica que todos los pasos del camino amoroso implican un movimiento horizontal en tanto que pertenecen a la esfera de los particulares, y que en cambio el ascenso ocurre únicamente con la contemplación de lo bello en sí (pp. 67-69). En este sentido, el autor establece que existe un «hiato» entre el camino y su conclusión que lo horizontal no puede cruzar, con lo cual el ascenso vertical solo se produce cuando la mente se ha ejercitado y fortalecido lo suficiente para dar un salto hasta lo que es en sí. Contra Chen, nosotros proponemos aquí que existe un carácter vertical que se puede encontrar en las distintas instancias horizontales del camino erótico. 
en esta misma instancia - llevar a cabo una producción espiritual: «Es preciso que quien avance correctamente [...] primero se enamore de un único cuerpo y engendre en él bellos razonamientos» (210a-b). El adverbio griego traducido por «en él» es entaũtha, "en este lugar», "aquí». Es decir que en el primer paso el encuentro con el otro cuerpo no es un acercamiento meramente sexual y sensible sino que desarrolla con la procreación de bellos argumentos también su aspecto vertical ascendente. Pero es precisamente la existencia de la horizontalidad que nunca deja de estar presente la que vuelve manifiesta la necesidad de un constante desvío en la continuidad vertical del ascenso, obligando al camino a tornarse una y otra vez hacia la inmanencia y la cotidianeidad del mundo que nos rodea.

Lo que me interesa plantear aquí es que, a partir de esta consideración, lo horizontal y lo vertical, la tierra y el cielo, lo humano y lo divino, dejan de oponerse como dos direcciones extremadamente diferentes y pasan a formar parte de una misma figura, con lo que Platón propone un dualismo que une sus partes antes que separarlas en polos opuestos y distintos. Son varias las ocasiones en que el diálogo plantea polos opuestos. En el comienzo, cuando los presentes se están acomodando en sus lugares, leemos, por ejemplo, de parte de Sócrates, la enunciación del primer par, lo lleno y lo vacío:

¡Qué bueno sería, Agatón, si la sabiduría fuese algo tal que con solo rozarnos fluyera del más lleno al más vacío de los dos; como el agua de las copas que fluye de la más llena a la más vacía a través de una hebra de lana! (175d)

Así también encontramos lo Vulgar y lo Celestial (180d), lo sano y lo enfermo (186b), la plenitud y la vacuidad (186c), lo unido y lo separado (190d), lo duro y lo blando (195d), lo mortal y lo inmortal (208b). Incluso en la misma estructura del diálogo, Platón coloca a un extremo - en los primeros discursos- al Amor como un dios, y en el otro - con el elogio de Alcibíades - como un hombre particular: Sócrates. En el medio, el Amor aparece como un daímon, puente entre lo humano y lo divino, que por su naturaleza siempre está moviéndose entre la sabiduría y la ignorancia, y los demás extremos. Cuando Sócrates pregunta qué poder tiene el Amor, Diotima le responde con lo siguiente: 
Interpreta y transmite a los dioses las cosas humanas y a los seres humanos las cosas de los dioses; de unos, las súplicas y sacrificios; y de los otros, las órdenes y las recompensas por los sacrificios. Como está en medio de ambos, rellena el intervalo, de modo que la totalidad esté estrechamente unida consigo misma. (202e)

Ya en el discurso de Erixímaco el grandísimo poder del Amor aparece trayendo «armonía» y «concordancia» a las cosas que difieren entre sí; o en el discurso de Aristófanes el Amor es el «médico de asuntos cuya cura haría surgir la mayor felicidad para la humanidad» (189d), esto es, la unión de las cuerpos mutilados y separados.

Con todo esto observamos cómo, al tiempo en que se manifiestan los extremos, Platón busca la manera de mostrarnos una forma de unión y reconciliación, es decir, manifestar cómo en realidad estos extremos están siempre juntos, y hay que considerarlos así porque, como pone en boca de Erixímaco, ambos están presentes (187e). De la misma forma ocurre en el ser humano, considerado como un compuesto de alma y cuerpo, y por esto mismo ninguna de las partes debería ser despreciada. En este camino interpretativo destacamos las lecturas de Fierro (2013) y Casertano (1997), quienes consideran que el alma se encuentra de hecho involucrada ${ }^{15}$ con el cuerpo, por lo cual la separación no es algo dado sino más bien algo a lograr. La responsabilidad de este trabajo le cae al alma, quien debe erigirse como dueña del cuerpo y pasar a experimentarlo como atravesado por sus propios deseos y elecciones. Esto implica una actuación de la filosofía en la vida aquí y ahora, donde despreciar al cuerpo quiere decir más bien trascenderlo y transformarlo de impedimento en vehículo de expresión de la actividad inteligente del alma. Broadie (2001), a su vez, sostiene que el alma está íntimamente conectada con el cuerpo, por ser al mismo tiempo alma intelecto y alma animadora. Para la autora, lo que primariamente involucra al alma con el cuerpo es el deseo de vivir, circunstancia en la que puede permanecer ajena a la posibilidad de existir como puro intelecto. Cornelli (2016), por su parte, afirma que Platón no propone un dualismo sustancialista, y que por el contrario en sus escritos queda abierto si el alma es algo inmaterial o no. Cornelli llega

15 El término que se utiliza en Phd. $66 \mathrm{~b}$ es sympephyrméne: "hecha un revoltijo», "entrelazada», «confundida». 
a afirmar que el alma humana puede y quiere a menudo tomar formas sensibles y somatizarse $\mathrm{e}^{16}$, asumir rasgos corpóreos $\mathrm{y}$, por su naturaleza camaleónica, asociarse con el mundo sensible; y que de hecho el alma se encarna de manera activa, queriendo hacer uso del cuerpo para poder conocer el mundo a través de los sentidos.

Buscaré mostrar ahora dónde se puede encontrar este doble aspecto horizontal-vertical del camino del amor en las diversas voces del diálogo. En los primeros cinco oradores, el aspecto horizontal aparece claramente en el deseo sexual y de acercamiento de los cuerpos. Tanto Fedro como Pausanias y Aristófanes consideran el Amor desde la perspectiva del encuentro amado-amante. Erixímaco considera además en particular el amor en el cuerpo desde su técnica médica, desde donde propone el cuidado armónico de sus partes. Agatón por su parte busca la mesura de los deseos corporales a través de la sensatez. Así leemos, por ejemplo, en esta instancia:

Yo, al menos no puedo señalar un bien mayor para un joven recién llegado a la adolescencia que un amante virtuoso, ni bien mayor para un amante que un jovencito amado. Pues la familia no logra inspirar tan bien como Eros ese sentimiento que necesariamente debe guiar a los hombres que durante toda la vida intentan vivir bien - ni tampoco los honores, el dinero ni ninguna otra cosa-. (178c-d. Discurso de Fedro)

En suma, tanto los dioses como los hombres conceden total libertad al amante. Así lo establece la norma de aquí. Cualquiera que reflexionara sobre esto podría suponer, efectivamente, que en esta ciudad se juzga que amar y trabar amistad con los amantes es algo enteramente bello. (183c. Discurso de Pausanias)

Zeus mudó entonces sus genitales adelante y con esto logró que la procreación tuviera lugar entre ellos (es decir a través del macho en la hembra), para que, de este modo, si un hombre se encontraba con una mujer, al entrelazarse, pudieran engendrar y la estirpe, subsistir. $Y$ a la vez para que, si un hombre se encontraba con otro, alcanzaran al menos cierta

${ }^{16}$ Cf. Phd. 79c donde, por ejemplo, se describe el estado de embriaguez del alma. 
saciedad en la unión y pudieran descansar y volver a sus actividades y ocuparse de los demás asuntos de la vida. (191c-d. Discurso de Aristófanes)

Pero en todos estos primeros elogios puede hallarse también el aspecto vertical del transitar amoroso, en tanto el deseo es utilizado en vistas a la virtud, y en definitiva, la felicidad. Así, Fedro vincula al Amor directamente con «la posesión de virtud y felicidad para los hombres tanto mientras viven como cuando han muerto» (180b). Erixímaco señala asimismo que el Amor «nos provee toda la felicidad» (188d), así como Pausanias considera el amar correctamente algo maravilloso (183c) y completamente bello porque se dirige hacia la virtud (185b). La misma idea reaparece luego en el discurso de Aristófanes como el fin de los asuntos amorosos - cuando leemos que "De los dioses, [el Amor] es el que más quiere a los hombres; es su protector y el médico de esas enfermedades por cuya curación el género humano gozaría de la más plena dicha» (189d) - , y en el discurso de Agatón describiendo al propio Amor como el dios más feliz (195a). Este movimiento hacia la virtud y la felicidad que se reitera en todos los primeros discursos puede considerarse ascendente o vertical, puesto que de hecho coincide con el fin de la scala amoris que en el discurso de Sócrates-Diotima se desarrolla de una manera más elaborada. Allí, igualmente, el fin de los asuntos amorosos es el momento más feliz, como leemos: «En este trance, querido Sócrates, más que en cualquier otro momento, la vida de un ser humano es digna de ser vivida - dijo la extranjera de Mantinea-, cuando contempla lo bello en sí» (211d).

En particular, se pueden encontrar estos aspectos horizontal y vertical más desarrolladamente en los discursos de Aristófanes y Alcibíades, personajes con quien - como ya hemos mostrado- Platón llama especialmente nuestra atención. Mostraré entonces brevemente cómo se replica la forma doble del peldaño en estos dos discursos.

En el caso del discurso de Aristófanes, el aspecto horizontal del peldaño es aquel que representa la primera consigna del primer paso descripto por Diotima amar un único cuerpo- y el aspecto vertical es la segunda consigna de este paso engendrar allí bellos argumentos. Entendemos con esto que el relato del poeta 
representa o evoca el primer paso de la scala amoris, siendo la búsqueda de la otra mitad el aspecto inmanente del amor horizontal, y la búsqueda de otro que concuerde naturalmente con uno en perspectivas el aspecto vertical que guiará al amante a la virtud y la felicidad.

Es en el amor por la otra mitad que aparece la necesidad externa, el andar horizontal e inmanente del amante. Aquí es donde más gráficamente el discurso de Aristófanes manifiesta la necesidad del desvío en el ascenso erótico: en la necesidad de salir a buscar al otro para retornar a un estado original. Aristófanes nos sumerge en un relato profundamente corporal en el que la búsqueda de la felicidad del ser humano es relatada como la búsqueda de la otra mitad del cuerpo de cada individuo. Después de que Zeus divide los perfectos cuerpos humanos, las mitades carentes andan en una constante búsqueda de su otra parte, anhelando el reencuentro con su propio fragmento, con su otro simbólico (191d). Badiou $(2003,2012)$ explica la necesidad de lo múltiple en términos de una experiencia de la diferencia, lo que él llama la vivencia del mundo desde la «escena del Dos». La interpretación de Badiou sin embargo, detiene el movimiento en el dos, en tanto que considera que el amor se vive en la multiplicidad y para la multiplicidad, y que lo que se busca es construir la verdad del Dos, y nunca la fusión en el Uno, «porque el amor, al fin y al cabo, sucede en el mundo» (Badiou, 2012, p. 36). Es decir que Badiou entiende el camino del amor como un transitar siempre horizontal e inmanente. El problema es que si nos quedamos varados/as en la escena del Dos, nuestro amor vagará siempre en la llanura, allí donde todos los reflejos rebotan siempre dentro del límite de la humanidad. Y el relato de Aristófanes, si bien se puede insertar en el ámbito horizontal del mundo, nos invita a descubrir un aspecto vertical de la búsqueda que sirve como resorte para continuar ascendiendo.

En cuanto al aspecto vertical, observamos que este surge en la problematización de la pretensión amorosa y la razón de la unión con el otro. Cuando los amantes se encuentran con su otro fragmento, ocurre lo siguiente:

Estos son los que pasan toda la vida juntos; sin siquiera saber qué esperan obtener uno del otro. Pues a ninguno se le ocurriría que se trata 
simplemente de los placeres de la unión sexual; es decir, que es solo por el deleite físico que el uno goza al unirse al otro con tanto ardor. (192c-d)

Las almas, aun deseosas, pretenden algo inefable que no pueden sino plantear oscuramente. Es aquí donde precisamente se manifiesta el aspecto vertical de este discurso. Lo que el alma desea es la verdadera unidad. Es por ello ninguno se negaría ante la supuesta oferta de Hefesto de fundir y fusionar las dos mitades en lo mismo, de modo que siendo dos lleguen a ser uno (192e). Esta unidad representa en el relato de Aristófanes la cura de la humanidad que hará surgir la mayor felicidad. Como señalábamos, el discurso de Aristófanes remite también a la segunda parte del primer paso de la iniciación de Diotima (engendrar bellos argumentos), completando con esto el primer peldaño. Aristófanes admite la gran dificultad que implica que cada individuo encuentre a su otra mitad y es por ello que en la búsqueda, recomienda no unirse momentáneamente a cualquier cuerpo, sino ir al encuentro de un otro con el que se compartan carácter y aspiraciones. En efecto, leemos: «Si esto es lo mejor, necesariamente lo será también lo que en la situación actual más se acerque a ello. Esto es, dar con un amado que por su mentalidad comparta la misma naturaleza que uno» (193c). En el relato de Aristófanes entonces, hallar un acuerdo espiritual o mental, más de carácter que de coincidencia física, es la manera presente que más se acerca a aquel estado de unidad del que el humano gozaba en su antigua naturaleza. Es decir que si bien la separación es gráficamente física (Zeus seccionando a los cuerpos humanos en dos) la implicancia de este quiebre esconde trazos de mayor profundidad que colaborarán a que el amante avance también «hacia arriba».

Finalmente, Alcibíades será la última voz en recordarnos lo sinuoso que resulta el camino del amor. En este caso, hallamos el aspecto horizontal en el amor apasionado de Alcibíades por un individuo particular (Sócrates), y la consideración de su propia belleza corpórea con la cual cree que conquistará al filósofo; y el aspecto vertical en la apreciación de la belleza más sutil del amado, su inteligencia: Alcibíades ve la belleza intelectual de quien pretende que sea su amante, y se maravilla y enloquece con sus palabras y argumentos: 
Yo, por mi parte, señores, si no fuera por el hecho de que van a creer que estoy perfectamente borracho, podría contarles, bajo juramento, qué he experimentado y qué experimento todavía hoy en día a causa de las palabras de este hombre. Cuando las escucho, mi corazón se acelera todavía más que el de los coribantes y mis lágrimas de derraman por causa de sus palabras. (215e)

Pero incluso, más allá de su elogio a Sócrates, el mismo personaje de Alcibíades es en Banquete el "necesario desvío». Como el cuerpo que irrumpe a los gritos, Alcibíades representa la horizontalidad en su mayor esplendor: es la belleza física que nos atrae coronada con hiedras y violetas, el joven celoso y enamorado que nos muestra lo mundano y lo cotidiano, el amor de todos los días con todas sus complicaciones y enredos. Como apunta Soares (2009, p. 103): «Alcibíades introduce en su discurso un punto de vista que restituye al término del diálogo la dimensión inmanente y temporal que, en pos de la trascendencia, había sido sacrificada en el discurso de Sócrates-Diotima».

De esta forma Aristófanes y Alcibíades nos revelan el necesario trazo horizontal de los peldaños ascendentes de la escalera del amor. El Banquete no abre la posibilidad de una meditación interior y solitaria que acerque al amante a la Belleza en sí. No hay una vía directa, como pudiera sugerir más adelante Plotino, y en cambio Platón nos indica que es necesario salirse de uno mismo y amar a otro. Este «otro» no es solo el amado en cuerpo y alma, sino también todas las cosas bellas del mundo: los pensamientos y las ideas, los buenos hábitos, las leyes y los conocimientos. Como indica Ludueña $(2015$, p. 82 ), si bien el camino amoroso comienza y culmina en singularidad, "es necesaria la mediación». No por nada la revelación de la verdad acerca de los asuntos amorosos es traída por una voz ajena a todo el resto: Diotima, femenina y extranjera, es también seña de la otredad y la diferencia. El necesario desvío horizontal en el ascenso implica salirse de uno mismo, ir a buscar la «escena del Dos» y actuar en ella para escalar cada vez más alto; significa hacer de la juntura de los 
ombligos del vientre una base sólida para elevarse desde el verdadero ombligo, el del cráneo ${ }^{17}$.

\section{El desapego}

Para concluir, sugeriré ahora que el necesario desvío o constante retorno al nivel horizontal de los cuerpos puede entenderse a partir de la noción de «desapego». Entiendo por «desapego» el resultado práctico de una comprensión que lleva a debilitar los lazos obsesivos que el amante tiene con el objeto de su amor. Esta noción atraviesa todo el movimiento de ascenso de la scala amoris y la propondré en lugar de la de «desprecio» que muchas veces ha llevado a la (mal)interpretación de que en el discurso de Sócrates-Diotima se plantea un rechazo a todo nivel corporal. El «desapego» en cambio libera al amante de una relación particularísima tanto física como mental, y le otorga la posibilidad de seguir avanzando en su camino amoroso. En lo que sigue mostraré cómo funciona esta noción en las distintas etapas de la iniciación de Diotima.

El cuerpo es el claro protagonista de los primeros dos peldaños de la escalera del amor. El primer paso dicta amar un único cuerpo, el segundo insta a descubrir la misma belleza igualmente en todos los cuerpos bellos ${ }^{18}$. El segundo peldaño, en tanto posterior, se describe como un momento de superación del primero. El amante, al descubrir que la belleza del cuerpo amado es la misma que la del resto de los cuerpos bellos, se vuelve amante de todos los cuerpos bellos (210b). En este momento se suele interpretar que Diotima, encarando un camino puramente intelectual, sugiere el desprecio de aquel anterior único cuerpo amado. Esta idea de desprecio puede confundirse con la creencia de que aquí Platón está despreciando el cuerpo, en un camino que debilitará cada vez más lo sensible hasta su completo rechazo. Sin embargo, el texto deja claro que esta nueva disposición que se entiende como desprecio no es hacia el cuerpo en sí mismo sino hacia el amor o deseo "de uno solo»

\footnotetext{
${ }^{17}$ En Ti. 90a, Platón describe la imagen del ser humano como una planta celeste que se eleva hacia la familia divina desde la cúspide de su cuerpo erguido.

${ }^{18}$ Es destacable al respecto el estudio de Konstan (2012), el cual nos sugiere no olvidar el íntimo vínculo entre la belleza y lo erótico a la hora de situarnos en la Grecia antigua, sobre todo porque la recepción moderna de los ideales estéticos clásicos despreció este sentido del término kállos ligado al éros.
} 
$(\text { enós })^{19}$. En otras palabras, lo que se ve superado en este peldaño es aquella instancia de mayor ignorancia en la que se creía que la belleza solo estaba en el único cuerpo amado. Podemos notar esto con mayor claridad en la comparación con distintas traducciones del mismo pasaje:

Y tras entender esto, es preciso que se convierta en amante de todos los cuerpos bellos y que deje pasar (chalásai) ese vehemente deseo por un solo cuerpo, no haciendo caso (kataphronésanta ${ }^{20}$ ) de él, es decir, dándole poca importancia (smikròn). (210b; traducción de Ludueña, 2015)

Si tiene esto en mente, se constituye en amante de todos los cuerpos bellos y debilita (chalásai) mucho el de uno solo despreciándolo (kataphronésanta) y considerándolo menor (smikròn)» (210b; traducción de Mársico)

And then realise for himself that the beauty that there is in any body whatever is the twin of that in any other, and if one should pursue beauty of outward form, it's quite mad not to regard the beauty in all bodies, and relax (chalásai) this passionate love for one body, despising (kataphronésanta) it and considering it a slight thing (smikròn). (210b; traducción de Rowe, 1998)

Si bien Rowe y Mársico eligen la idea de «desprecio» para traducir el verbo griego kataphronéo, mientras que Ludueña evita este término ${ }^{21}$, es evidente que lo

\footnotetext{
${ }^{19}$ Nótese que Diotima explica que es preciso constituirse pántọn tỗn kalōn somáton erastés ("amante de todos los cuerpos bellos»), donde pánton tỗn kalōn somáton ("de todos los cuerpos bellos») aparecen en genitivo. En el mismo caso está la palabra inmediatamente posterior enós («de uno solo»), seguida por la partícula adversativa dé («pero», «en cambio»). Con lo cual lo que se está contraponiendo es el carácter de sujeto enamorado, en tanto su amor se despierte a partir de todos los cuerpos bellos, o de uno solo. Este último caso es el que será superado en esta instancia.

20 Vid. LSJ, s. v. «kataphronéö: A, «look down upon», «think slightly of». Si bien en A, 2 leemos que el verbo significa "regard slightly, despise», esto no necesariamente tiene que entenderse como un desprecio en el sentido de "rechazo absoluto». En realidad, la idea de "desprecio» implica aquí un reconocimiento del menor valor que tiene aquello que "se mira desde arriba», es decir, desde un estadio superior de mayor comprensión. Cf. Platón Ap. 28c, donde leemos que Aquiles desestima el peligro (toũ kindúnou katephrónesen). En este contexto, Aquiles conocía que debía morir inmediatamente después de Héctor y es por eso que lo vemos actuar dándole poco valor o no haciendo caso al peligro y a la muerte.

${ }^{21}$ La elección de Ludueña («no haciendo caso») va de la mano con su decisión de traducir el primer término - chaláõ - como «dejar pasar». Me parece acertada la consideración de Ludueña (2015, p.189) de que la idea aquí no es "combatir» el deseo sino «dejarlo ir tal y como vino» (Vid. LSJ, s. v. "chaláõ», donde otras acepciones de las ya mencionadas sobre este término son: I, 2. let fall, let go, 3. release), pues el deseo de hecho surge, y la tarea del amante que avanza bien guiado es no volverse esclavo de este deseo particular, sino ver que la belleza se extiende igualmente en el resto de los cuerpos bellos. No hay diferencias relevantes en lo que refiere al tercer término (smikrós).
} 
que se considera menor (una vez que el amante se da cuenta que la belleza presente en el resto de los cuerpos es la misma) es el deseo por un solo cuerpo. En ningún caso, incluso aunque se utilice la idea de desprecio, lo que se rechaza es el cuerpo en sí mismo, o el amor dirigido a los cuerpos como objeto de deseo. Tras esta superación, el amante se desapega de la situación de deseo a un único cuerpo, relaja este amor especial y transforma su visión volviéndola más amplia. Es así como pasa a considerar menor su experiencia previa en la que solo podía ver la belleza en el cuerpo de su amado especial. La superación en este sentido no implica tampoco que el amante pasará a considerar feo el cuerpo de su amado o despreciable su amor por su enamorado particular, sino que va a pasar a considerar este deseo único como una posibilidad limitada. Por eso, si pensamos esta nueva disposición como un «desapego» en lugar de un «desprecio» o "rechazo», entenderemos más transparentemente un cambio de percepción que genera una relación más relajada o desinteresada con el cuerpo del otro, esto es, una nueva forma de amor.

El siguiente paso en el camino amoroso es considerar más valiosa la belleza en las almas que la belleza en los cuerpos, de modo que aunque el cuerpo tenga una mínima belleza, si el amante encuentra razón en el amado, con eso se satisface y ama, procreando argumentos y haciéndose mejor para ver también luego la belleza en las ocupaciones y las leyes y los conocimientos. Este escalón permite un ascenso del todo relevante, pues conlleva la comprensión que trasciende el nivel de lo físico. Lo que me interesa subrayar aquí es que la noción de «desapego» sigue teniendo sentido, puesto que no ocurre solamente en el nivel del cuerpo sino que se aplica también a lo intangible.

En una primera instancia, el amante que ya se ha desapegado de la relación especial con un único cuerpo, desliga ahora su deseo de todo lo pesado, en la comprensión de que no es necesaria una forma física para la apreciación de la belleza. Entonces, pasa a considerar «que es algo menor lo bello según el cuerpo» (210c). Nuevamente, esto no significa que se deje de relacionar con los cuerpos ni las cosas bellas del mundo; por el contrario, seguirá haciéndolo, pero de una forma cada vez más libre. A medida que la Belleza se deja ver en un escenario cada vez más vasto, el 
progresivo desapego a todo lo corporal vuelve cada vez más ligero el andar del amante. Llegado este punto, observamos que el desapego ocurre también en el ámbito de lo no corporal:

Al fijarse de ahí en más en la variedad de lo bello y ya no solo en la belleza de un único ser (como un vulgar criado que, negligente y de escaso carácter, contento con la belleza de un único muchachito, de algún hombre o hábito de vida, vive en esclavitud), se vuelve hacia el vasto mar de lo bello. (210d)

Apegarse a cualquier cosa bella, sea un cuerpo o un argumento, un hábito o una ocupación particular y creer que esa cosa es especialmente bella, no hace más que esclavizar al amante a una experiencia limitada. Es necesario, pues, transformar todo amor particular y amar cada vez más libremente, lo cual implica desapegarse no solo de la belleza de un muchacho o de un hombre (i.e. del cuerpo) sino también de un hábito de vida (i.e. de todo aquello no-corporal que se ame especialmente). Este es el nivel en el que el amante «da a luz muchos bellos y magníficos razonamientos y pensamientos en un generoso amor por la sabiduría (philosophía)»(210d). Es preciso que el filósofo transforme sus vínculos amorosos deshaciendo todo deseo particular y erigiéndose como verdadero amante de la sabiduría, pues solo así estará preparado para contemplar una belleza que ya no se representará en ninguna otra cosa, sino que se mostrará en sí misma bella y causa de lo bello en todas las otras cosas.

\section{Conclusión}

A través del análisis del camino amoroso del Banquete, puede concluirse que el modelo antropológico que Platón presenta en este diálogo utiliza positivamente tanto al alma como al cuerpo para la búsqueda filosófica guiada por el amor. En el Banquete el aspecto sensible se desarrolla como una noción plena de sentido que es necesario rastrear con cautela en todos los personajes, no solo en sus discursos sino también en sus movimientos y acciones dramáticas.

Como hemos mostrado, Platón nos hace señas para que reparemos en la importancia de la horizontalidad que dobla el camino ascendente. Un ataque de hipo, 


\section{Sain Abril}

desorden corporal que solo se irá con un movimiento aún más desordenado, el estornudo, evoca el primer paso en el ascenso erótico: amar a un único cuerpo y engendrar allí bellos argumentos. La aparición desordenada y estrepitosa de un cuerpo bello, entre gritos, música, colores y vino, nos saca del ensimismamiento del banquete y nos lleva a la vida cotidiana, allí donde efectivamente se da el amor a los cuerpos, y allí donde de hecho se puede iniciar el camino del amor. Mientras que el discurso de Sócrates-Diotima nos enseña la manera correcta (orthõs) de iniciarse en los asuntos del amor - un camino de ascenso vertical-, Platón subraya en varios otros momentos, sobre todo con Aristófanes y Alcibíades, la necesidad de un desvío, que la sacerdotisa extranjera no deja de incluir, pero que Platón cree necesario resaltar, para que no se olvide. El cuerpo, en este esquema, representa el motor del movimiento; en palabras de Fierro (2019), "es el activador natural del deseo por la belleza y en tal sentido constituye un instrumento precioso para iniciar la búsqueda de la verdad» (p. 192).

Finalmente, la noción de «desapego» nos permitió aprehender la posibilidad de un camino a la vez horizontal y vertical, que asciende a lo divino sin dejar de apoyarse en la inmanencia y sin rechazar los previos peldaños de su andar. El desapego libera al amante de una relación particularísima y especial, y le otorga en cambio la posibilidad de expandir su amor libremente. Esto incluye al cuerpo en un panorama en el que ya no se malinterpreta su desprecio aislado como un rechazo a todo lo corporal, sino que se lo coloca a la par de cualquier otro objeto bello, corpóreo o incorpóreo, que pueda resultar una obsesión limitante para el amante. De esta forma, el amante avanzará en su camino comprendiendo gradualmente la universalidad de la belleza, y así, en el sexo, la gastronomía, el arte y la política, deberá estar atento a no volverse esclavo de un cuerpo, una idea, un hábito o un pensamiento, sino continuar expandiendo su amor utilizando cada experiencia de deseo como posibilidad de elevarse al encuentro con lo divino. 


\section{Bibliografía}

ANNAS, J. (2003). Plato. A Very Short Introduction. Oxford University Press.

BAILLY, A. (1950). Dictionnaire Grec-Francais. Paris: Hachette.

BADIOU, A. (2003). «Filosofía y amor», en Condiciones. Siglo XXI. México, pp. 239-259.

(2012). Elogio del amor. Buenos Aires: Paidós.

BOERI, M.D. (2016). "Éros y synousía en el Simposio», en M. Tulli \& M. Erler (Eds.), Plato in Symposium. Selected Papers from the Tenth Symposium Platonicum (pp. 362-370). Sankt Agustin: Akademia Verlag.

BRANDWOOD, L. (1976). A word index to Plato. Leeds: W.S. Maney.

BROADIE, S. (2001), Soul and Body in Plato and Descartes en Proceedings of the Aristotelian Society. New Series. Vol. 101, pp. 295-308.

BROCHARD, V. (1940). "Sobre el Banquete de Platón», en Estudios sobre Sócrates y Platón. Buenos Aires.

BURNET, I. (1899-1906). Platonis Opera, 5 vols., Oxford: Oxford University Press.

CASERTANO, G. (1997). II (in) nome di Eros. Una lettura del discorso di Diotima nel Simposio platonico. Elenchos, XVIII, pp. 277-310.

CHANTRAINE, P. (1974). Dictionnaire étymologique de la langue grecque. Histoire des mots. Paris. (=DELG).

CHEN, L. C. H. (1983). «Knowledge of Beauty in Plato's Symposium», en The Classical Quarterly, New Series, Vol. 33, No. 1, pp. 66-74. Cambridge University Press.

CORNELLI, G. (2016). «A alma-camaleão e sua plasticidade: dualismos platônicos no Fédon», Archai 16: 127-138.

CORNFORD, F. M. (1974). «La doctrina del éros en el Banquete de Platón» (1974), en La filosofía no escrita. Barcelona: Editorial Ariel.

CORTÁZAR, J. (1962). Instrucciones para subir una escalera. Buenos Aires: Alfaguara.

DIXSAUT, M. (2001). Le naturel philosophe - essai sur les dialogues de Platon. Paris: Librairie Philosophique J. Vrin.

DOVER, K. (1980). Plato. Symposium, edition with introduction and commentary. Cambridge. 
FERRARI, G. R. F. (1987). Listening to the Cicadas: A study of Plato's Phaedrus. Cambridge. Cambridge University Press.

FIERRO, M. A. (2013). «Alma encarnada - cuerpo amante en el Fedón de Platón», en Benítez Grobet, L. \& Velázquez Zaragoza, A. (eds.), en Platonismo y neoplatonismo en la modernidad filosófica. Mexico DF: 25-58. "Loving and lovable bodies in Plato's Symposium», XI Symposium Platonicum: The «Symposium». Proceedings II, International Plato Society Universidad de Pisa, Departamento de Filología, Literatura y Lingüística, Pisa, Italia, 2013*: 258-262.

(2019). «Amor carnal, amor platónico en el Banquete», en Estud.filos $\mathrm{N}^{\circ}$ 59, Universidad de Antioquia, pp. 189-219.

GIERSE, G. (1970). «Zur Komposition des platonischen Simposion», Gymnasium 77, pp. 49-76.

GUTHRIE, W. K. C. (1975). A History of Greek Philosophy. Cambridge.

HOFFMANN, E. (1947) Über Platons Symposioum. Heildelberg.

HOLMES, B. (2010). The symptom and the subject. The emergence of the physical body in Ancient Greece. Princeton and Oxford: Princeton University Press.

ISENBERG, M. W. (1940). The order of the discourses in Plato's Symposioum. Chicago.

KONSTAN, D. (2012). El concepto de belleza en el mundo antiguo y su recepción en Occidente. Nova Tellus, 30, pp. 133-147. ISSN: 0185-3058. Disponible en: https://www.redalyc.org/articulo.oa?id=591/59128312005.

LIDDELL, H. G., Scott, R., Jones, H. S., \& McKenzie, R. (1940). A Greek-English Lexicon. Oxford: Clarendon Press. (= LSJ)

LUDUEÑA, E. (2015). Platón, Banquete. Traducción, introducción y notas. Buenos Aires: Colihue.

MÁRSICO, C. (2009). Platón, Banquete. Buenos Aires: Miluno. ROWE, C. J. (1998). Plato. Symposium, edited with an Introduction, Translation and Commentary. Oxford: Aris \& Phillips Classical Texts.

NUSSBAUM, M. C. (2001) [1986]. The Fragility of Goodness. Luck and Ethics in Greek Tragedy and Philosophy. Cambridge: Cambridge University Press. 
PEIXOTO, M. C. D. (2012) «Corpo, anima, visibile, invisibile nel Simposio platonico», Marino, S. (trad.), en Borges de Araujo, Jr. y Cornelli, G., II Simposio di Platone: un banchetto di interpretazioni. Napoli: Lofredo Editore, pp. 159-179.

REALE, G. (2010), «La concepción del hombre» en Reale, G. y Antiseri, D., Historia del Pensamiento Filosófico y Científico. Tomo I. Antigüedad y Edad Media. Barcelona: Herder.

ROBIN, L. (1908). La théorie platonicienne de l'amour. París: Félix Alcan Éditeur.

ROSEN, S. (1968). Plato's Symposioum. Londres: New Haven.

ROWE, C. J. (1998). Plato. Symposium, edited with an Introduction, Translation and Commentary. Oxford: Aris \& Phillips Classical Texts.

SOARES, L. (2012). «La erótica platónica en perspectiva. Notas para una lectura del Banquete - Estudio preliminar», en Mársico, C. (trad.), Platón, Banquete, Miluno, Buenos Aires, pp. 13-128.

VELÁZQUEZ, O. «En torno a "súbitamente" en el Banquete de Platón», en Revista Chilena de Literatura, No. 27/28 (Apr. - Nov., 1986), pp. 67-76.

VERNANT, J. -P. (2001). El individuo, la muerte y el amor en la Antigua Grecia. Barcelona: Paidós.

VIGO, A. (2009). Platón. Fedón. Buenos Aires: Colihue. 